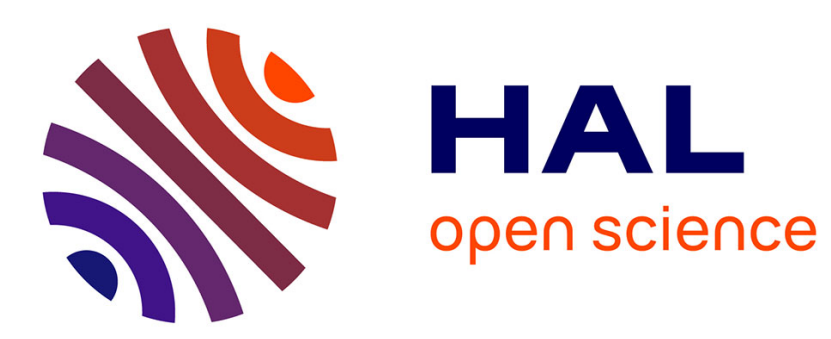

\title{
Bounded Control for DC/DC Converters: Application to Renewable Sources
}

\author{
Alessio Iovine, Frédéric Mazenc
}

\section{To cite this version:}

Alessio Iovine, Frédéric Mazenc. Bounded Control for DC/DC Converters: Application to Renewable Sources. CDC 2018 - 57th IEEE Conference on Decision and Control, Dec 2018, Miami, United States. hal-01918305

\section{HAL Id: hal-01918305 \\ https://hal.inria.fr/hal-01918305}

Submitted on 10 Nov 2018

HAL is a multi-disciplinary open access archive for the deposit and dissemination of scientific research documents, whether they are published or not. The documents may come from teaching and research institutions in France or abroad, or from public or private research centers.
L'archive ouverte pluridisciplinaire HAL, est destinée au dépôt et à la diffusion de documents scientifiques de niveau recherche, publiés ou non, émanant des établissements d'enseignement et de recherche français ou étrangers, des laboratoires publics ou privés. 


\title{
Bounded Control for DC/DC Converters: Application to Renewable Sources
}

\author{
Alessio Iovine $^{1}$, Frédéric Mazenc $^{2}$
}

\begin{abstract}
Advanced control methods are nowadays increasing in interest for power electronics devices. This paper proposes a nonlinear control law for a DC/DC boost converter dedicated to extract the maximum power from a photovoltaic (PV) array, taking into account the constraints of the control action. System stability analysis is provided by a proper Lyapunov function. Simulations on SimPowerSystems validate how the developed control strategy is able to properly control the converter with good performances.
\end{abstract}

Index Terms-Bounded control, Nonlinear control, Control for Power Converters

\section{INTRODUCTION}

Nowadays, the utilization of complex control techniques for regulating power converters is becoming a common standard. Indeed, the technological progress and its rise in complexity of the required tasks, together with an always increasing demand of better performance, requires more advanced control methods. As a result, the development and application of control techniques different from the classic Proportional Integral Derivative (PID) for power converters is a well established research field, and its related literature is continuously enriched [1], [2], [3], [4], [5], [6], [7], [8], [9], [10], [11].

Although the variety of advanced control results for DC/DC converters, only few of them take into account the physical limitations of the systems and how they impact on the performances and the operating region [4], [9], [11], [12], [13]. To develop an unbounded control law for systems for which large values for the control are prohibited, is quite accepted in literature. This is due to the complexity of the problem of constructing bounded feedbacks. As a consequence, the bounds are taken into account in an empiric way for selecting the gains of the control law and to try to provide an estimation of the operating region of the system related to the acting disturbances and the control law.

When constraints on the control input are taken into account, the used control methods have some drawbacks: for example, calculation time due to the Linear Matrix Inequalities (LMI) methods (see [4], [12], [13]) or dynamics cancellation due to backstepping (see [9]) can generate a high error in the control with respect to small measurement errors.

In this paper, a bounded nonlinear control design is used to correctly control a boost DC/DC converter connected to

\footnotetext{
${ }^{1}$ Efficacity, R\&D Institute for Urban Energy Transition, Paris, France. (e-mail: a.iovine@efficacity.com).

2 Inria EPI DISCO, L2S-CNRS-CentraleSupélec, 3 rue Joliot Curie, 91192, Gif-sur-Yvette, France

(e-mail: frederic.mazenc@12s.centralesupelec.fr).
}

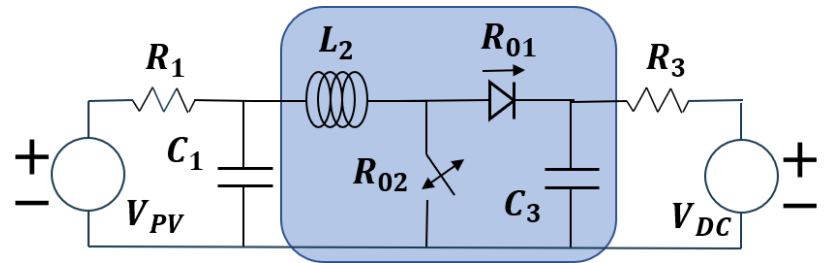

Fig. 1. The adopted electrical scheme. The boost DC/DC converter is introduced in the blue rectangular region, and is connected to a strong DC grid and to the PV array.

a photovoltaic (PV) panel. Our objective is similar to the one of [14]: ensuring stability and at the same time the satisfaction of some performance requirements. However, it is worth mentioning that the design we propose does not rely on the main result of [14] nor on classical techniques of constructions of bounded control laws, such as the ones of [15], [16], [17]. The equilibrium point of the system is provided by a Maximum Power Point Tracking (MPPT) algorithm, which is supposed to be placed in a higher hierarchical control scheme [18]. The used control technique imposes a desired equilibrium point to the systems, respecting the bounds of the control law while imposing a certain desired convergence rate. Such technique allows for an online calculation of the control input, increasing system performances. Moreover, the problem of nonminimum phase of the boost converter does not affect the introduced control law.

The paper is organized as follows. Section II introduces the considered model, while Section III describes preliminary properties of the system. In Section IV the control law is developed, and Section V outlines simulations to validate it. Conclusions are provided in Section VI.

\section{Modeling}

The considered model for a DC/DC converter connected to a DC bus and a PV source is taken from [19]. The equivalent circuit representation for a DC/DC boost converter connected to a strong DC grid and the PV source can be expressed using a state space average model, under the hypothesis to then use a Pulse Width Modulation (PWM) technique for control purposes [20], [21]. Three state variables are needed for the system model: the capacitor voltages $V_{C_{1}}: \mathbb{R} \rightarrow \mathbb{R}^{+}$ and $V_{C_{3}}: \mathbb{R} \rightarrow \mathbb{R}^{+}$( $x_{1}$ and $x_{3}$ respectively) and the inductor current $I_{L_{2}}: \mathbb{R} \rightarrow \mathbb{R}\left(x_{2}\right)$. Let $X(t)=\left(x_{1}(t), x_{2}(t), x_{3}(t)\right)$. In the remaining part of the paper, time dependence will be omitted due to lack of space. $C_{1}, C_{3}, R_{1}, R_{3}, L_{2}, R_{01}, R_{02}$ are known positive values of the capacitors, resistances and the inductor while $V_{D C} \in \mathbb{R}^{+}$is the constant grid voltage. 


\begin{tabular}{||c|c|c|c||}
\hline \hline Parameter & Value & Parameter & Value \\
\hline$a_{1}$ & $\frac{1}{R_{1} C_{1}}$ & $a_{2}$ & $\frac{1}{C_{1}}$ \\
\hline$a_{3}$ & $\frac{1}{L_{2}}$ & $a_{4}$ & $\frac{R_{01}}{L_{2}}$ \\
\hline$a_{5}$ & $\frac{1}{R_{3} C_{3}}$ & $a_{6}$ & $\frac{1}{C_{3}}$ \\
\hline \hline
\end{tabular}

TABLE I

THE POSITIVE VALUES OF THE PARAMETERS.

The PV source is here represented as a voltage source, which is piecewise constant; $V_{P V} \in \mathbb{R}^{+}$is the photovoltaic panel voltage. Fig. 1 depicts the considered framework. The measured signals are the states $x_{1}, x_{2}, x_{3}$ and the PV array and DC grid constant voltages $d_{1}=V_{P V}, d_{2}=V_{D C}$. $v=1-u$ is the control input, which is defined as the duty cycle of the circuit. The electrical scheme in Fig. 1 can then be modeled as follows:

$$
\left\{\begin{array}{l}
\dot{x}_{1}=-a_{1} x_{1}-a_{2} x_{2}+a_{1} d_{1}, \\
\dot{x}_{2}=a_{3} x_{1}-a_{3} x_{3} v-a_{4} x_{2}, \\
\dot{x}_{3}=-a_{5} x_{3}+a_{6} x_{2} v+a_{5} d_{2} .
\end{array}\right.
$$

Here $x, v, d_{1}, d_{2}$ are measurable. The parameters' value are depicted in Table I. It must to be noticed that the system has the property to have 2 time-scales, since it satisfies the motion separation principle derived from singular perturbation theory [22]. Furthermore, to have feasible solutions, the disturbances are such that $d_{1}>0, d_{2}>0, d_{2}>d_{1}$.

The control input is bounded as follows:

$$
v \in[0,1] .
$$

\section{A. Equilibrium point}

Let us determine the equilibrium point of the system (1) with $v \equiv v_{*}$ where $v_{*}>0$ is a constant. Let $E_{*}=$ $\left(x_{1 *}, x_{2 *}, x_{3 *}\right)$ denote an equilibrium point. Hence

$$
\begin{aligned}
& x_{1 *}=\left(\frac{a_{6} v_{*}^{2}}{a_{5}}+\frac{a_{4}}{a_{3}}\right) a_{1} \frac{d_{1}-v_{*} d_{2}}{a_{1}\left(\frac{a_{6} v_{*}^{2}}{a_{5}}+\frac{a_{4}}{a_{3}}\right)+a_{2}}+v_{*} d_{2}, \\
& x_{2 *}=a_{1} \frac{d_{1}-v_{*} d_{2}}{a_{1}\left(\frac{a_{6} v_{*}^{2}}{a_{5}}+\frac{a_{4}}{a_{3}}\right)+a_{2}}, \\
& x_{3 *}=\frac{a_{6} v_{*}}{a_{5}} a_{1} \frac{d_{1}-v_{*} d_{2}}{a_{1}\left(\frac{a_{6} v_{*}^{2}}{a_{5}}+\frac{a_{4}}{a_{3}}\right)+a_{2}}+d_{2} .
\end{aligned}
$$

It follows that the equilibrium point $E_{*}$ is positive if the following inequality is satisfied:

$$
v_{*}<\frac{d_{1}}{d_{2}} .
$$

\section{PRELIMINARY ANALYSis}

Let us consider a constant $v_{*} \geq 0$ (not fixed yet, such that condition (4) is satisfied). As a consequence, the system in (1) admits an equilibrium point $E_{*}=\left(x_{1 *}, x_{2 *}, x_{3 *}\right)$ with $x_{3 *}>0$.

The common used technique when dealing with power systems is the one of creating a fast inner loop dealing with the current ( $x_{2}$ in this case) and an outer control loop dealing with the desired voltage, to be used as a reference for the current loop. This because the system satisfies the separation principle conditions, even if such conditions are not really used when developing the control law. In this work, a similar approach is used: the current $x_{2}$ will be the directly controlled variable, and the other two voltages will react as a consequence. To this purpose, some preliminary analysis has to be done about the voltage $x_{3}$ in order to test its boundedness, and to allow to focus for the control law development just on the other dynamics.

\section{A. Boundedness of $x_{3}$}

Now, let us perform a change of feedback: $v=v_{*}+\mu$ and impose on $\mu$ to be such that $|\mu|_{\infty} \leq \bar{\mu}$, where $\bar{\mu}>0$ is to be defined later. Noticing that $e_{1}=x_{1}-x_{1 *}, e_{2}=x_{2}-x_{2 *}$, $e_{3}=x_{3}-x_{3 *}$ satisfy

$$
\left\{\begin{array}{l}
\dot{e}_{1}=-a_{1} e_{1}-a_{2} e_{2}, \\
\dot{e}_{2}=a_{3} e_{1}-a_{3} v_{*} e_{3}-a_{4} e_{2}-a_{3} \mu(t)\left(e_{3}+x_{3 *}\right), \\
\dot{e}_{3}=-a_{5} e_{3}+a_{6} v_{*} e_{2}+a_{6} \mu(t)\left(e_{2}+x_{2 *}\right) .
\end{array}\right.
$$

Let us consider the positive definite quadratic function:

$$
V\left(e_{1}, e_{2}, e_{3}\right)=\frac{a_{3} a_{6}}{2} e_{1}^{2}+\frac{a_{2} a_{6}}{2} e_{2}^{2}+\frac{a_{2} a_{3}}{2} e_{3}^{2} .
$$

Its time derivative is

$$
\begin{aligned}
\dot{V}(t)= & -a_{1} a_{3} a_{6} e_{1}^{2}-a_{2} a_{4} a_{6} e_{2}^{2}-a_{2} a_{3} a_{5} e_{3}^{2}+ \\
& -a_{2} a_{3} a_{6} x_{3 *} e_{2} \mu(t)+a_{2} a_{3} a_{6} x_{2 *} e_{3} \mu(t) .
\end{aligned}
$$

As an immediate consequence,

$$
\begin{aligned}
\dot{V} \leq & -a_{1} a_{3} a_{6} e_{1}^{2}-a_{2} a_{4} a_{6} e_{2}^{2}-a_{2} a_{3} a_{5} e_{3}^{2}+ \\
& +a_{2} a_{3} a_{6}\left|x_{3 *}\right|\left|e_{2}\right| \bar{\mu}+a_{2} a_{3} a_{6}\left|x_{2 *}\right|\left|e_{3}\right| \bar{\mu} .
\end{aligned}
$$

Notice that it follows that if $\bar{\mu}=0$, then $E_{*}$ is GES Considering

$$
\varsigma=\min \left\{a_{1}, a_{4}, a_{5}\right\}
$$

and the equalities in (3), we obtain

$$
\begin{aligned}
\dot{V} \leq & -\varsigma V+a_{6} \frac{\sqrt{2 a_{2} a_{3}} a_{1} d_{1}}{a_{1} \frac{a_{4}}{a_{3}}+a_{2}} \bar{\mu} \sqrt{V}+ \\
& +a_{3} \sqrt{2 a_{2} a_{6}}\left(\frac{a_{1} a_{6}}{a_{5}} \frac{d_{1}}{a_{1} \frac{a_{4}}{a_{3}}+a_{2}}+d_{2}\right) \bar{\mu} \sqrt{V} .
\end{aligned}
$$

Let $\epsilon \in\left(0, \frac{1}{2}\right)$ be any constant. As an immediate consequence, there is a value $t_{\star} \geq 0$ such that for all $t \geq t_{*}$,

$$
\sqrt{V} \leq(1+\epsilon) \sqrt{2} \sqrt{a_{2} a_{3}} \frac{c_{1}}{\varsigma} \bar{\mu}
$$

where

$$
c_{1}=\sqrt{a_{3} a_{6}}\left(\frac{a_{1} a_{6}}{a_{5}} \frac{d_{1}}{a_{1} \frac{a_{4}}{a_{3}}+a_{2}}+d_{2}\right)+a_{6} \frac{a_{1} d_{1}}{a_{1} \frac{a_{4}}{a_{3}}+a_{2}} .
$$

From the definition of $V$, it follows that

$$
\sqrt{\frac{a_{2} a_{3}}{2}}\left|e_{3}\right| \leq(1+\epsilon) \sqrt{2} \sqrt{a_{2} a_{3}} \frac{c_{1}}{\varsigma} \bar{\mu} .
$$

It follows that

$$
\left|x_{3}(t)-x_{3 *}\right| \leq 2(1+\epsilon) \frac{c_{1}}{\varsigma} \bar{\mu} .
$$


Thus

$$
x_{3 *}-2(1+\epsilon) \frac{c_{1}}{\varsigma} \bar{\mu} \leq x_{3}(t) \leq x_{3 *}+2(1+\epsilon) \frac{c_{1}}{\varsigma} \bar{\mu} .
$$

We deduce that when

$$
2(1+\epsilon) \frac{c_{1}}{\varsigma} \bar{\mu} \leq \epsilon x_{3 *}
$$

then

$$
(1-\epsilon) x_{3 *} \leq x_{3}(t) \leq(1+\epsilon) x_{3 *} .
$$

Considering (3) and (12) in (16), it has to be remarked that it is possible to take the following expression for $\bar{\mu}$,

$$
\bar{\mu}=\frac{\epsilon}{2(1+\epsilon)} d_{2} \frac{\varsigma}{c_{1}}
$$

which is independent from $v_{*}$. Furthermore, due to (18), select a $v_{*}>0$ such that

$$
\frac{\epsilon v_{*}}{1-\epsilon}<\bar{\mu}
$$

implies to satisfy also the following inequality:

$$
v_{*}<d_{2} \frac{\varsigma}{2 c_{1}} \text {. }
$$

\section{B. Change of control for the $\left(x_{1}, x_{2}\right)$-subsystem}

Now, let us focus our attention on the $\left(x_{1}, x_{2}\right)$-subsystem of (1):

$$
\left\{\begin{array}{l}
\dot{x}_{1}=-a_{1} x_{1}-a_{2} x_{2}+a_{1} d_{1}, \\
\dot{x}_{2}=a_{3} x_{1}-a_{3} x_{3} v-a_{4} x_{2} .
\end{array}\right.
$$

Let $\psi$ be defined as follows:

$$
\psi(s)=\left\{\begin{array}{cl}
(1-\epsilon) x_{3 *} & s \leq(1-\epsilon) x_{3 *}, \\
s & s \in\left[(1-\epsilon) x_{3 *},(1+\epsilon) x_{3 *}\right], \\
(1+\epsilon) x_{3 *} & s \geq(1+\epsilon) x_{3 *} .
\end{array}\right.
$$

The inequality (19) ensures that there is $\bar{\nu}>0$ such that

$$
\frac{1}{1-\epsilon} \bar{\nu}+\frac{\epsilon\left|v_{*}\right|}{1-\epsilon} \leq \bar{\mu} .
$$

Notice for later use that this inequality rewrites as

$$
\bar{\nu} \leq(1-\epsilon) \bar{\mu}-\epsilon v_{*} .
$$

Now, let us perform the following change of feedback

$$
v=\frac{x_{3 *} \nu}{\psi\left(x_{3}\right)}
$$

with $\nu$ such that $\left|\nu-v_{*}\right| \leq \bar{\nu}$.

Then, the control input $v(t)$ can be rewritten as

$$
\frac{x_{3 *} \nu(t)}{\psi\left(x_{3}(t)\right)}=v(t)=v_{*}+\mu(t)
$$

and consequently $\mu(t)$ results

$$
\mu(t)=\frac{x_{3 *} \nu(t)}{\psi\left(x_{3}(t)\right)}-v_{*} .
$$

With the following calculations

$$
\begin{aligned}
\mu(t) & =\frac{x_{3 *}\left(\nu(t)-v_{*}\right)}{\psi\left(x_{3}(t)\right)}+\frac{x_{3 *} v_{*}}{\psi\left(x_{3}(t)\right)}-v_{*} \\
& =\frac{x_{3 *}\left(\nu(t)-v_{*}\right)}{\psi\left(x_{3}(t)\right)}+\frac{x_{3 *}-\psi\left(x_{3}(t)\right)}{\psi\left(x_{3}\right)} v_{*}
\end{aligned}
$$

and the possibility to write

$$
\left|\frac{x_{3 *}-\psi\left(x_{3}\right)}{\psi\left(x_{3}\right)} v_{*}\right| \leq \frac{\epsilon x_{3 *}}{(1-\epsilon) x_{3 *}} v_{*}=\frac{\epsilon v_{*}}{1-\epsilon},
$$

the boundedness of $\mu(t)$ with respect to its maximum value $\bar{\mu}$ is proven, as in (23):

$$
|\mu(t)| \leq \frac{x_{3 *} \bar{\nu}}{\psi\left(x_{3}\right)}+\frac{\epsilon v_{*}}{1-\epsilon} \leq \frac{\bar{\nu}}{1-\epsilon}+\frac{\epsilon v_{*}}{1-\epsilon} \leq \bar{\mu} .
$$

Then, the $\left(x_{1}, x_{2}\right)$-subsystem of (1) becomes:

$$
\left\{\begin{array}{l}
\dot{x}_{1}=-a_{1} x_{1}-a_{2} x_{2}+a_{1} d_{1}, \\
\dot{x}_{2}=a_{3} x_{1}-a_{3} x_{3} \frac{x_{3 *} \nu}{\psi\left(x_{3}\right)}-a_{4} x_{2},
\end{array}\right.
$$

and from (30) and the analysis in Section III-A ,we deduce that, when $t \geq t_{\star}$, then $x_{3} \in\left[(1-\epsilon) x_{3 *},(1+\epsilon) x_{3 *}\right]$. It follows that for all $t \geq t_{\star}$, the $\left(x_{1}, x_{2}\right)$-subsystem can be written as

$$
\left\{\begin{array}{l}
\dot{x}_{1}=-a_{1} x_{1}-a_{2} x_{2}+a_{1} d_{1}, \\
\dot{x}_{2}=a_{3} x_{1}+b \nu-a_{4} x_{2}
\end{array}\right.
$$

where $b=-a_{3} x_{3 *}$.

\section{Feedback stabilization}

\section{A. Control law design}

Let us now rewrite the subsystem in (32) with respect to its equilibrium point, defining $\tilde{x}_{i}=x_{i}-x_{i *}, i=1,2$. Also, let us perform the following change of feedback:

$$
\omega=b \nu+a_{3} x_{1 *}-a_{4} x_{2 *} .
$$

It results in

$$
\left\{\begin{array}{l}
\dot{\tilde{x}}_{1}=-a_{1} \tilde{x}_{1}-a_{2} \tilde{x}_{2}, \\
\dot{\tilde{x}}_{2}=a_{3} \tilde{x}_{1}-a_{4} \tilde{x}_{2}+\omega,
\end{array}\right.
$$

where

$$
|\omega| \leq a_{3} x_{3 *} \bar{\nu}
$$

because

$$
b \nu+a_{3} x_{1 *}-a_{4} x_{2 *}=-a_{3} x_{3 *}\left(\nu-v_{*}\right)
$$

and $\left|\nu-v_{*}\right| \leq \bar{\nu}$.

To facilitate the control design, we introduce $\xi$ as

$$
\xi=\tilde{x}_{2}-\rho \frac{k \tilde{x}_{1}}{1+k^{3}\left(\tilde{x}_{1}^{2}+\tilde{x}_{2}^{2}\right)}
$$

where $\rho$ is a small tuning positive parameter and $k$ a positive gain, and calculate its dynamical behavior:

$$
\dot{\xi}=a_{3} \tilde{x}_{1}-a_{4} \xi+\eta
$$

with

$$
\begin{aligned}
\eta & =-a_{4}\left(\rho \frac{k \tilde{x}_{1}}{1+k^{3}\left(\tilde{x}_{1}^{2}+\tilde{x}_{2}^{2}\right)}\right)+\left(1+\frac{2 \rho k^{3} \tilde{x}_{1} \tilde{x}_{2}}{\left[1+k^{3}\left(\tilde{x}_{1}^{2}+\tilde{x}_{2}^{2}\right)\right]^{2}}\right) \omega \\
& +\rho k \frac{\left[a_{1} \tilde{x}_{1}+a_{2} \tilde{x}_{2}\right]\left[1+k^{3}\left(\tilde{x}_{2}^{2}-\tilde{x}_{1}^{2}\right)\right]+2 k^{2} \tilde{x}_{1} \tilde{x}_{2}\left[a_{3} \tilde{x}_{1}-a_{4} \tilde{x}_{2}\right]}{\left[1+k^{3}\left(\tilde{x}_{1}^{2}+\tilde{x}_{2}^{2}\right)\right]^{2}} .
\end{aligned}
$$

Let us introduce the positive tuning parameter $h$ and define the saturation function $\operatorname{sat}_{1}(m)$ as

$$
\operatorname{sat}_{1}(m)=\left\{\begin{array}{cl}
m & |m| \leq 1 \\
\frac{m}{|m|} & m>1
\end{array}\right.
$$


Now we select

$$
\eta=-\operatorname{ssat}_{1}\left(\frac{k}{h} \xi\right)
$$

and obtain

$$
\left\{\begin{aligned}
\dot{\tilde{x}}_{1} & =-a_{1} \tilde{x}_{1}-a_{2} \rho \frac{k \tilde{x}_{1}}{1+k^{3}\left(\tilde{x}_{1}^{2}+\tilde{x}_{2}^{2}\right)}-a_{2} \xi \\
\dot{\xi} & =a_{3} \tilde{x}_{1}-a_{4} \xi-\operatorname{hsat}_{1}\left(\frac{k}{h} \xi\right)
\end{aligned}\right.
$$

For this system, we can achieve locally arbitrarily fast speed of convergence by choosing $k$ sufficiently large and we can do this with a feedback $\omega$ which respects the needed size constraint.

Then the formula of $v$ is

$$
\begin{aligned}
v= & \frac{-a_{3} x_{1 *}+a_{4} x_{2 *}}{b \frac{\psi\left(x_{3}\right)}{x_{3 *}}}-\frac{h s a t_{1}\left(\frac{k}{h} \xi\right)}{b \frac{\psi\left(x_{3}\right)}{x_{3 *}}\left(1+\frac{2 \rho k^{3} \tilde{x}_{1} \tilde{x}_{2}}{\left[1+k^{3}\left(\tilde{x}_{1}^{2}+\tilde{x}_{2}^{2}\right)\right]^{2}}\right)}+ \\
& +\frac{a_{4}\left(\rho \frac{k \tilde{x}_{1}}{1+k^{3}\left(\tilde{x}_{1}^{2}+\tilde{x}_{2}^{2}\right)}\right)}{b \frac{\psi\left(x_{3}\right)}{x_{3}}\left(1+\frac{2 \rho k^{3} \tilde{x}_{1} \tilde{x}_{2}}{\left[1+k^{3}\left(\tilde{x}_{1}^{2}+\tilde{x}_{2}^{2}\right)\right]^{2}}\right)}+ \\
& -\frac{\rho k \frac{\left[a_{1} \tilde{x}_{1}+a_{2} \tilde{x}_{2}\right]\left[1+k^{3}\left(\tilde{x}_{2}^{2}-\tilde{x}_{1}^{2}\right)\right]+2 k^{2} \tilde{x}_{1} \tilde{x}_{2}\left[a_{3} \tilde{x}_{1}-a_{4} \tilde{x}_{2}\right]}{\left[1+k^{3}\left(\tilde{x}_{1}^{2}+\tilde{x}_{2}^{2}\right)\right]^{2}}}{b \frac{\psi\left(x_{3}\right)}{x_{3 *}}\left(1+\frac{2 \rho k^{3} \tilde{x}_{1} \tilde{x}_{2}}{\left[1+k^{3}\left(\tilde{x}_{1}^{2}+\tilde{x}_{2}^{2}\right)\right]^{2}}\right)} .
\end{aligned}
$$

\section{B. Stability analysis}

We analyze the stability properties of the system (42) via the positive definite function:

$$
V\left(\tilde{x}_{1}, \xi\right)=\frac{1}{2 a_{2}} \tilde{x}_{1}^{2}+\frac{1}{2 a_{3}} \xi^{2} .
$$

Its derivative along the trajectories of the system (42) is

$$
\begin{aligned}
\dot{V}(t)= & \frac{1}{a_{2}} \tilde{x}_{1}\left(-a_{1} \tilde{x}_{1}-a_{2} \rho \frac{k \tilde{x}_{1}}{1+k^{3}\left(\tilde{x}_{1}^{2}+\tilde{x}_{2}^{2}\right)}-a_{2} \xi\right)+ \\
& +\frac{1}{a_{3}} \xi\left(a_{3} \tilde{x}_{1}-a_{4} \xi-h s a t_{1}\left(\frac{k}{h} \xi\right)\right) \\
= & -W\left(\tilde{x}_{1}, \xi\right)
\end{aligned}
$$

with

$$
\begin{aligned}
W\left(\tilde{x}_{1}, \xi\right)= & \frac{1}{a_{2}} a_{1} \tilde{x}_{1}^{2}+\rho \frac{k \tilde{x}_{1}^{2}}{1+k^{3}\left(\tilde{x}_{1}^{2}+\tilde{x}_{2}^{2}\right)}+ \\
& +\frac{1}{a_{3}} a_{4} \xi^{2}+\frac{1}{a_{3}} h s a t_{1}\left(\frac{k}{h} \xi\right) \xi .
\end{aligned}
$$

Since $W$ is definite positive, we deduce that the origin of the system is globally asymptotically stable. Besides, $W$ is approximated in a neighborhood of the origin by $\left(\frac{a_{1}}{a_{2}}+\rho k\right) \tilde{x}_{1}^{2}+\frac{a_{4}+k}{a_{3}} \xi^{2}$. We deduce that the speed of convergence can be increased by increasing the constant $k$.

\section{Selected equilibrium point}

To highlight the generality of the introduced controller and the possibility to apply it to the boost converter, a further remark is needed. Indeed, the equilibrium point in (3), $x_{*}$, is function of the value of the control at the equilibrium, $v_{*}$, i. e. $x_{*}=f\left(v_{*}\right)$. Since in reality the available degree of freedom is used to impose an equilibrium, while the equilibrium for the other dynamics is calculated as a consequence of it, it must be remarked that the previous introduced analysis still holds when fixing the equilibrium according to a desired reference, with just the hypothesis to consider equilibria that are in the admissible set $\Omega_{*}$. The possibilities are:

\begin{tabular}{||c|c|c|c||}
\hline \hline Parameter & Value & Parameter & Value \\
\hline$R_{1}$ & $100 \mathrm{~m} \Omega$ & $R_{3}$ & $100 \mathrm{~m} \Omega$ \\
\hline$C_{1}$ & $100 \mathrm{~m} F$ & $C_{3}$ & $10 \mathrm{~m} F$ \\
\hline$L_{2}$ & $33 \mathrm{mH}$ & $R_{01}$ & $10 \mathrm{~m} \Omega$ \\
\hline \hline
\end{tabular}

TABLE II

THE VALUES OF THE PARAMETERS.

- a value $x_{1}^{e}$ is imposed;

$$
\begin{aligned}
& x_{1 *}=x_{1}^{e} \\
& x_{2 *}=\frac{a_{1}}{a_{2}}\left(d_{1}-x_{1}^{e}\right) \\
& x_{3 *}=\frac{1}{2}\left[d_{2}+\sqrt{d_{2}^{2}+4 \beta_{1} \phi_{1}\left(x_{1}^{e}-\gamma_{1} \phi_{1}\right)}\right],
\end{aligned}
$$

where $\beta_{1}=\frac{a_{6} a_{1}}{a_{5} a_{2}}, \gamma_{1}=\frac{a_{4} a_{1}}{a_{3} a_{2}}, \phi_{1}=\left(d_{1}-x_{1}^{e}\right)$.

- a value $x_{2}^{e}$ is imposed;

$$
\begin{aligned}
& x_{1 *}=d_{1}-\frac{a_{2}}{a_{1}} x_{2}^{e} \\
& x_{2 *}=x_{2}^{e} \\
& x_{3 *}=\frac{1}{2}\left[d_{2}+\sqrt{d_{2}^{2}+4 \frac{a_{6}}{a_{5}} x_{2}^{e}\left[d_{1}-\gamma_{2} x_{2}^{e}\right]}\right],
\end{aligned}
$$

where $\gamma_{2}=\left(\frac{a_{2}}{a_{1}}+\frac{a_{4}}{a_{3}}\right)$.

Without loss of generality, it must to be remarked that similar calculations apply also in the case where $x_{3}^{e}$ is imposed.

\section{Simulations}

The simulations are developed in a realistic electric framework, which is SimPowerSystem, a MatlabR2016b toolbox. Both the situations described in Section IV-C are considered, in order to show the effectiveness of the proposed control law either when fixing $x_{1}^{e}$ or $x_{2}^{e}$. The value of the parameters are introduced in Table II. The considered simulation time is about 5 seconds, the discrete simulation step is of $0.01 \mathrm{~ms}$ and the frequency for the PWM is $20 \mathrm{kH}$.

The fact to use a realistic toolbox for electrical systems results in a more complex framework, since the considered hypothesis of fixed disturbances cannot be met in a real scenario. Nevertheless, the introduced control law is shown to properly perform even in case of slowly (with respect to the state variables) time-varying disturbances $d_{1}$ and $d_{2}$. The reference value for $x_{1}^{e}$ or $x_{2}^{e}$ is considered piecewise constant, and varying each one second; it is supposed to be provided by a MPPT algorithm, which is not modeled in this work.

\section{A. Case 1: choice of a value $x_{1}^{e}$}

Fig. 2 depicts both the reference $x_{1}^{e}$ and the dynamical behavior of the state variable $x_{1}$ when the system is controlled by the control input introduced in equation (43) with target to impose a desired $x_{1}^{e}$. As shown, the controller perfectly meets its target, since the error dynamics converges to zero. Furthermore, since the choice of the desired reference $x_{1}^{e}$ has been properly done, the controller fits its boundaries, as it is depicted in Fig. 5. The dynamical evolutions of the other two equilibria for $x_{2}$ and $x_{3}$, together with their dynamics, are introduced in Fig. 3 and 4: they are time varying since $d_{1}$ and $d_{2}$ are time varying. 


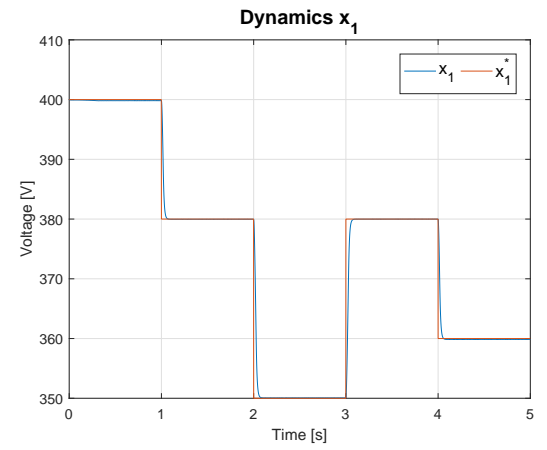

Fig. 2. Case 1: The dynamical behavior of $x_{1}$ with respect to its chosen reference $x_{1}^{e}$.

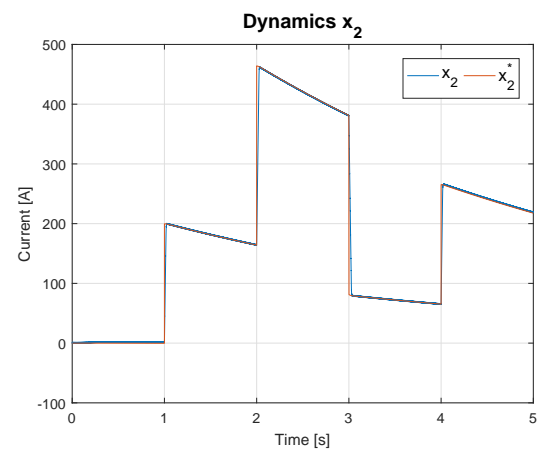

Fig. 3. Case 1: The dynamical behavior of $x_{2}$ with respect to its reference $x_{2}^{*}$, calculated as a consequence of the choice of $x_{1}^{e}$.

As already mentioned, the reference is piecewise constant and changes its value every seconds. Since the source is a PV panel, the reference is selected to provide only positive currents. The oscillations taking place in Fig. 4 are due to the switching nature of the implemented system, and the different way of representing them with the average model here used: nevertheless, it is easy to verify that $x_{3}$ follows its reference.

\section{B. Case 2: choice of a value $x_{2}^{e}$}

Here the reference $x_{2}^{e}$ is imposed, and the resulting controller implemented. As shown in Fig. 9, it results in a feedback that is bounded in its operating region. Fig. 6, 7

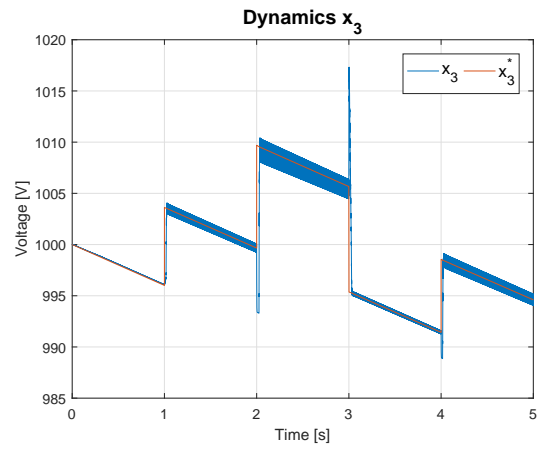

Fig. 4. Case 1: The dynamical behavior of $x_{3}$ with respect to its reference $x_{3}^{*}$, calculated as a consequence of the choice of $x_{1}^{e}$.

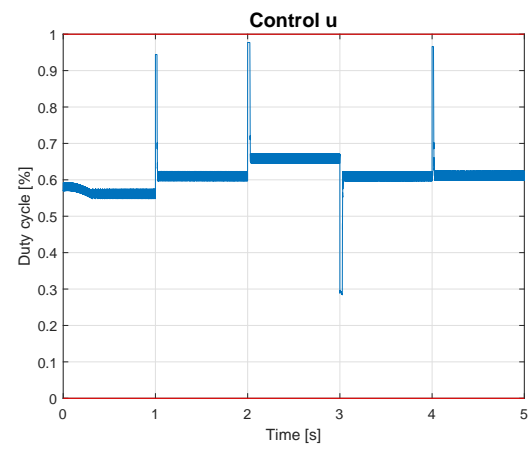

Fig. 5. Case 1: The dynamical behavior of the controller $u$ with respect to its boundaries, in the case of choice of $x_{1}^{e}$.

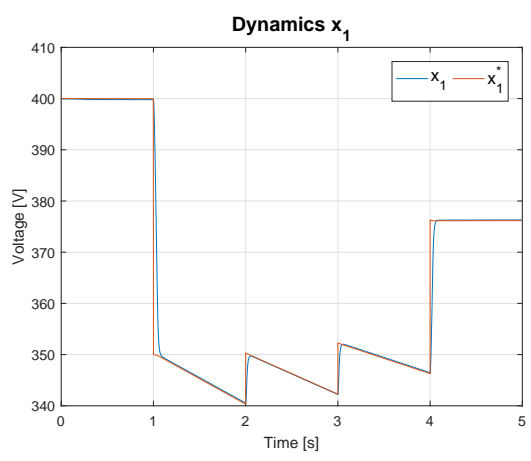

Fig. 6. Case 2: The dynamical behavior of $x_{1}$ with respect to its reference $x_{1}^{*}$, calculated as a consequence of the choice of $x_{2}^{e}$.

and 8 depict the dynamical behaviours of the state variables with respect to their equilibria: convergence is assured for all of them.

\section{Discussion on the implemented control}

From Fig. 5 and 9 it must be noted that the developed control action allows for a "smooth" curve in the obtained duty cycle. It is important since it results in less stress for the components and the devices.

This outcome is due to the proper choice of the parameters $\epsilon, \rho$ and $k$ with respect to the considered set $\Omega_{*}$ of the equilibria and a desired convergence rate. The resulting controller is shown to be bounded even when dealing with the transient due to the new reference, and not only in steady state.

The calculation of such set $\Omega_{*}$ is out of the scope of this paper. Future works will focus on an analytical description of this set and how to calculate it, as well as optimal choices of the aforementioned parameters.

\section{CONCLUSIONS}

In this work a new nonlinear control law for DC/DC boost converters connected to renewable power sources as PV is introduced. The control input is calculated online, and takes into account physical limitations while providing desired performances. Stability analysis is investigated by a proper Lyapunov function, and the system is shown to be asymptotically stable around the chosen equilibrium point. 


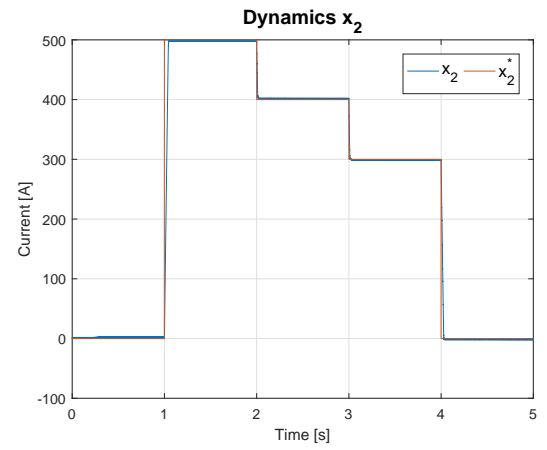

Fig. 7. Case 2: The dynamical behavior of $x_{2}$ with respect to its chosen reference $x_{2}^{e}$.

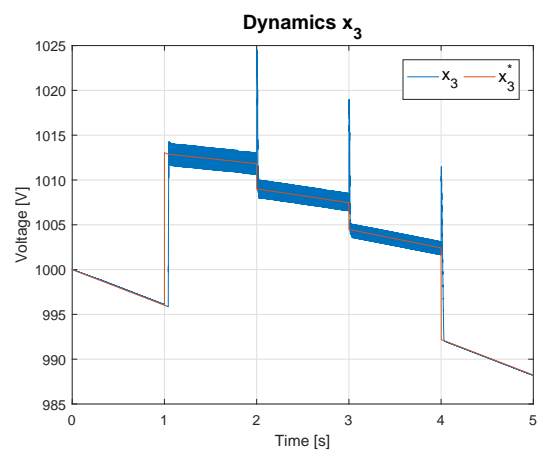

Fig. 8. Case 2: The dynamical behavior of $x_{3}$ with respect to its reference $x_{3}^{*}$, calculated as a consequence of the choice of $x_{2}^{e}$.

Realistic simulation results are given in SimPowerSystems to show the effectiveness of the proposed control action.

Future works will deal with the optimization of such control law with respect to the broadest set of possible equilibria.

\section{REFERENCES}

[1] H. J. Sira Ramirez and R. Silva-Ortigoza, Control design techniques in power electronics devices. Springer, 2006.

[2] S. Bacha, I. Munteanu, and A. I. Bratcu, Power electronic converters modeling and control. Springer, 2014.

[3] S. Mariethoz, S. Almer, M. Baja, A. Beccuti, D. Patino, A. Wernrud, J. Buisson, H. Cormerais, T. Geyer, H. Fujioka, U. Jonsson, C.Y. Kao, M. Morari, G. Papafotiou, A. Rantzer, and P. Riedinger, "Comparison of Hybrid Control Techniques for Buck and Boost DC-

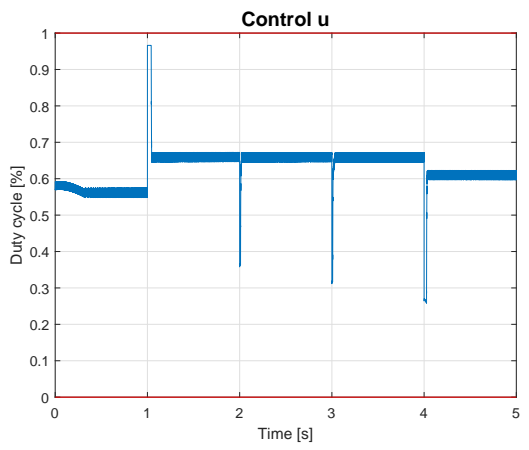

Fig. 9. Case 2: The dynamical behavior of the controller $u$ with respect to its boundaries, in the case of choice of $x_{2}^{e}$.
DC Converters," IEEE Transactions on Control Systems Technology, vol. 18, pp. 1126-1145, Sept 2010.

[4] C. Olalla, I. Queinnec, R. Leyva, and A. E. Aroudi, "Optimal StateFeedback Control of Bilinear DC/DC Converters With Guaranteed Regions of Stability," IEEE Transactions on Industrial Electronics, vol. 59, pp. 3868-3880, Oct 2012.

[5] S. Vazquez, J. I. Leon, L. G. Franquelo, J. Rodriguez, H. A. Young, A. Marquez, and P. Zanchetta, "Model predictive control: A review of its applications in power electronics," IEEE Industrial Electronics Magazine, vol. 8, pp. 16-31, March 2014.

[6] T. A. F. Theunisse, J. Chai, R. G. Sanfelice, and W. P. M. H. Heemels, "Robust global stabilization of the dc-dc boost converter via hybrid control," IEEE Transactions on Circuits and Systems I: Regular Papers, vol. 62, pp. 1052-1061, April 2015.

[7] G. C. Konstantopoulos and Q. C. Zhong, "Nonlinear control of DC/DC power converters with inherent current and power limitation," in 2016 24th Mediterranean Conference on Control and Automation (MED), pp. 949-954, June 2016.

[8] A. Iovine, S. B. Siad, G. Damm, E. De Santis, and M. D. Di Benedetto, "Nonlinear Control of a DC MicroGrid for the Integration of Photovoltaic Panels," IEEE Transactions on Automation Science and Engineering, vol. 14, pp. 524-535, April 2017.

[9] A. Hernandez-Mendez, J. Linares-Flores, H. Sira-Ramirez, J. F. Guerrero-Castellanos, and G. Mino-Aguilar, "A backstepping approach to decentralized active disturbance rejection control of interacting boost converters," IEEE Transactions on Industry Applications, vol. 53, no. 4, pp. 4063 - 4072, 2017.

[10] Y. M. Alsmadi, V. Utkin, M. A. Haj-ahmed, and L. Xu, "Sliding mode control of power converters: Dc/dc converters," International Journal of Control, vol. 0, no. 0, pp. 1-22, 2017.

[11] J. Moreno-Valenzuela and O. Garca-Alarcon, "On Control of a Boost DC-DC Power Converter under Constrained Inpu," Complexity, vol. 2017, Article ID 4143901, 2017.

[12] M. Tucci, S. Riverso, and G. Ferrari-Trecate, "Line-independent plugand-play controllers for voltage stabilization in dc microgrids," IEEE Transactions on Control Systems Technology, vol. PP, no. 99, pp. 1-9, 2017.

[13] C. Olalla, R. Leyva, A. E. Aroudi, and I. Queinnec, "Robust LQR Control for PWM Converters: An LMI Approach," IEEE Transactions on Industrial Electronics, vol. 56, pp. 2548-2558, July 2009.

[14] S. Tarbouriech and J. G. D. Silva, "Synthesis of controllers for continuous-time delay systems with saturating controls via LMIs," IEEE Trans. Aut. Contr., vol. 45, no. 1, pp. 105-111, 2000.

[15] A. Teel, "A nonlinear small gain theorem for the analysis of control systems with saturation," IEEE Trans. Aut. Contr., 1996.

[16] F. Mazenc and A. Iggidr, "Backstepping with bounded feedbacks.," Systems and Control Letters, vol. 51, no. 3/4, pp. 235-245, 2004.

[17] F. Mazenc and L. Praly, "Adding an integration and global asymptotic stabilization of feedforward systems," IEEE Trans. Aut. Contr., 1996.

[18] A. Iovine, G. Damm, E. De Santis, and M. D. Di Benedetto, "Management Controller for a DC MicroGrid integrating Renewables and Storages," in 20th IFAC World Congress on International Federation of Automatic Control (IFAC 2017), (Toulouse, France), 2017.

[19] G. Walker and P. Sernia, "Cascaded DC-DC converter connection of photovoltaic modules," IEEE Transactions on Power Electronics, vol. 19, pp. 1130-1139, July 2004.

[20] R. Middlebrook and S. Cuk, "A general unified approach to modelling switching-converter power stages," International Journal of Electronics, vol. 42, no. 6, pp. 521-550, 1977.

[21] A. Bindra, "Pulsewidth modulated controller integrated circuit: Four decades of progress [a look back]," IEEE Power Electronics Magazine, vol. 1, pp. 10-44, Sept 2014.

[22] H. K. Khalil, Nonlinear systems. Prentice Hall, 2002. 